\title{
The Lapse Function in Friedmann-Lemaître-Robertson-Walker Cosmologies
}

\author{
F. Melia* \\ Department of Physics, The Applied Math Program, \\ and Department of Astronomy, The University of Arizona, AZ 85721, USA
}

\begin{abstract}
The Friedmann-Lemaitre-Robertson-Walker (FLRW) metric, the backbone of modern cosmology, is founded on the cosmological principle, which assumes homogeneity and isotropy throughout the cosmos. One of its simplifications is the choice of lapse function $g_{t t}=1$, regardless of which stress-energy tensor $T^{\mu \nu}$ is used in Einstein's field equations. It is sometimes argued that this selection is justified by gauge freedom, given that $g_{t t}$ in FLRW may be a function solely of $t$, not of the spatial coordinates, permitting a redefinition of the time. We show in this paper, however, that the comoving frame in the Hubble expansion is non inertial for all but a few special cases of the expansion factor $a(t)$. Changing the gauge changes the frame of reference and cannot alter this property of the expansion profile, since it would of necessity reformat the metric using the coordinates of a non-comoving observer. We therefore suggest that the pre-selection of $g_{t t}=1$, independently of the equation of state in the cosmic fluid, incorrectly avoids the time dilation that ought to be present relative to the actual free-falling frame when $\ddot{a} \neq 0$.

PACS numbers: 04.20.-q, 04.20.Ex, 95.36.+x, 98.80.-k, 98.80.Jk
\end{abstract}

*John Woodruff Simpson Fellow; Electronic address: fmelia@email.arizona.edu 


\section{INTRODUCTION}

The Friedmann-Lemaittre-Robertson-Walker (FLRW) metric (Eq. 1 below) is the cornerstone of modern cosmology. Given its high degree of symmetry, based on the presumed isotropy and homogeneity of the Universe, it is a special, well-studied member of the general class of spherically-symmetric spacetimes describing systems undergoing gravitational collapse or expansion [1-4]. But for various historical reasons, the development of FLRW followed a different approach than that of the others [5]. Specifically, whereas the equations governing gravitational collapse are derived from Einstein's equations using a general form

of the metric coefficients, the Friedmann equations [6] describing the cosmic expansion are obtained after all the symmetries underlying the cosmological principle have been introduced to greatly simplify the FLRW metric prior to its insertion into the field equations - regardless of which equation of state (in terms of the total energy density $\rho$ and pressure $p$ ) is subsequently adopted for the stress-energy tensor $T^{\mu \nu}$.

Though subtle, this difference turns out to have a profound impact on how we describe the evolution of the Universe because, by fiat, we require the FLRW lapse function $g_{t t}$ to always be equal to 1, regardless of whether or not the cosmic expansion is accelerating. Already, there is a possible conflict with this choice because, even in his early work, Einstein realized that his theory of gravity correctly reduces to Newton's law in the weak-field limit only if $g_{t t}=1+2 \Phi / c^{2}$, where $\Phi$ is the gravitational potential. The implication of this is that if $g_{t t}=1$, the observer sees no acceleration and no time dilation.

This issue does not receive the attention it deserves, mainly because conventional wisdom has it that $g_{t t}$ in FLRW can-at most-be a function only of time, not space, for consistency with the cosmological principle, which is undoubtedly true. And therefore one can, if necessary, change the gauge $d t \rightarrow d t^{\prime} \equiv \sqrt{g_{t t}} d t$ so that the coordinate dependence of the lapse function again disappears from the metric. But there is always something suspicious about this procedure because, at the very heart of it, a change in gauge in general relativity (GR) is really a transformation of the coordinates, i.e., a change in the frames of reference [5]. Since the FLRW metric is, by construction, the spacetime in the comoving frame, where does the gauge transformation $d t \rightarrow d t^{\prime} \equiv \sqrt{g_{t t}} d t$ take us?

In this paper, we shall use the Local Flatness Theorem (sometimes referred to as the strong equivalence principle) in GR to prove that the choice of lapse function $g_{t t}=1$ is in 
fact valid only for two specific equations of state: $\rho=p=0$ (i.e., Minkowski space); and $\rho+3 p=0$ (the so-called zero active mass condition), which results in a Universe expanding at a constant rate i.e., $a(t) \propto t$ in Eq. (1) below.

\section{LOCAL FLATNESS THEOREM}

Let $x^{\mu}=(c t, x, y, z)$ be the coordinates in the co-moving frame, $g_{\mu \nu}$ the metric coefficients, and $\Gamma_{\mu \nu}^{\lambda}$ the corresponding Christoffel symbols. Throughout this paper, we assume spatial flatness, given that most of the observations today appear to be supporting this initial condition in the cosmic expansion [7]. Thus, the relevant FLRW metric may be written simply as

$$
d s^{2}=L(t) c^{2} d t^{2}-a(t)^{2}\left(d x^{2}+d y^{2}+d z^{2}\right),
$$

with an expansion factor $a(t)$ and metric coefficients

$$
g_{\mu \nu}=\left(\begin{array}{cccc}
L & 0 & 0 & 0 \\
0 & -a^{2} & 0 & 0 \\
0 & 0 & -a^{2} & 0 \\
0 & 0 & 0 & -a^{2}
\end{array}\right) .
$$

To demonstrate our point, we have generalized the metric by introducing the lapse function $g_{t t} \equiv L(t)$, allowing us to examine the constraints it must satisfy in order to solve Equation (4) below. As noted earlier, this simple functional form of $L$ is consistent with the assumption of isotropy and homogeneity in the cosmological principle, which permits only a dependence of the lapse function on time, but not space. It is straightforward to show that the corresponding non-zero Christoffel symbols in the comoving frame are

$$
\begin{aligned}
\Gamma_{00}^{0} & =\frac{1}{L} \frac{\partial L}{\partial c t} \\
\Gamma_{i i}^{0} & =\frac{1}{L c} a \dot{a} \\
\Gamma_{i 0}^{i} & =\Gamma_{0 i}^{i}=\frac{1}{c} \frac{\dot{a}}{a} .
\end{aligned}
$$

According to the Local Flatness Theorem [8], there exists a locally inertial coordinate frame $\xi^{\mu}(X)$ in the neighborhood of any spacetime point $X^{\mu}$ in $x^{\mu}$. The $\xi^{\mu}$ frame is in free fall at $X^{\mu}$, so any non inertial effects in the coordinate system $x^{\mu}$ - such as a time dilationcan be measured absolutely relative to $\xi^{\mu}$. These effects are absent only if the co-moving frame were itself in free fall, in which case it would be inertial to begin with. 
For convenience, and without loss of generality, let us place $X^{\mu}$ at the common origin of $x^{\mu}$ and $\xi^{\mu}$. Also, given the evident symmetry, it is safe to assume that $\xi^{0}$ may depend solely on $t$ and the radius $r=\sqrt{x^{2}+y^{2}+z^{2}}$, while $\xi^{i}$ may be a function only of $t$ and $x^{i}$ (with $i=1,2$ or 3$)$. The equations satisfied by $\xi^{\mu}$ are [8]

$$
\frac{\partial^{2} \xi^{\alpha}}{\partial x^{\mu} \partial x^{\nu}}=\Gamma_{\mu \nu}^{\lambda} \frac{\partial \xi^{\alpha}}{\partial x^{\lambda}}
$$

The $\alpha=\mu=\nu=0$ component in this expression thus yields the following relation that must be satisfied by the lapse function $L(t)$ in terms of $a(t)$ :

$$
\xi^{0}=f(r) \int^{c t} L\left(t^{\prime}\right) d\left(c t^{\prime}\right)+g(r)
$$

where $f(r)$ and $g(r)$ are functions yet to be determined. The $\alpha=\mu=0, \nu=i$ component gives

$$
\frac{\partial}{\partial x^{i}}\left(\frac{\partial \xi^{0}}{\partial c t}\right)=\frac{1}{c} \frac{\dot{a}}{a}\left[\frac{x^{i}}{r} \frac{d f}{d r} \int^{c t} L\left(t^{\prime}\right) d\left(c t^{\prime}\right)+\frac{x^{i}}{r} \frac{d g}{d r}\right] .
$$

Evidently, these two expressions show that $d g / d x^{i}=0$, and we may simply choose $g(r)=0$, given that it represents a constant temporal translation. Crucially, we find from Equations (5) and (6) that

$$
\int^{c t} L\left(t^{\prime}\right) d\left(c t^{\prime}\right)=c L(t) \frac{a}{\dot{a}} .
$$

The choice of lapse function $L(t)=1$ is therefore consistent only with the expansion factor

$$
a(t) \equiv\left(\frac{t}{t_{0}}\right)
$$

written in conventional form for a spatially flat metric using the current age, $t_{0}$, of the Universe.

Note, however, that Equation (4) is also trivially satisfied with $\xi^{0}=c t$ and $L(t)=1$ when $a=$ constant, in which case the spacetime curvature is identically zero, i.e., $\Gamma_{\mu \nu}^{\alpha}=0$. In other words, relative to a local inertial frame $\xi^{\mu}$ at $\vec{x}=0$, the lapse function in FLRW may be set equal to 1 only for the very special cases (1) $a=$ constant (i.e., Minkowski space) and $(2) a(t)=\left(t / t_{0}\right)$. For any other expansion factor (representing acceleration), the time $t$ in the comoving frame must necessarily be dilated relative to the time $\xi^{0}$ in the inertial frame.

If $L(t)=1$, and only then, the time coordinate (Eq. 5) in the local inertial frame may be written $\xi^{0}=f(r) c t$, in terms of a function $f(r)$ that we may now identify. Equation (4) 
provides us with one more relevant component, corresponding to $\alpha=0, \mu=\nu=i$ :

$$
\frac{\left(x^{i}\right)^{2}}{r^{2}} \frac{d^{2} f}{d r^{2}}+\frac{\left(x^{j}\right)^{2}+\left(x^{k}\right)^{2}}{r^{3}} \frac{d f}{d r}=\frac{1}{\left(c t_{0}\right)^{2}} f,
$$

where $i \neq j \neq k$. The solution to this equation, correct to second order in $r$, is

$$
f(r)=1+\frac{1}{2}\left(\frac{r}{c t_{0}}\right)^{2}
$$

and, recalling that $\xi^{\mu}$ are the coordinates in a locally inertial frame in the vicinity of $\vec{x}=0$, we see that $\xi^{0} \rightarrow c t$, as one would expect.

Turning now to the spatial coordinates, we see that according to Equation (4),

$$
\xi^{i}=h\left(x^{i}\right) \int^{c t} L\left(t^{\prime}\right) d\left(c t^{\prime}\right),
$$

in which the function $h\left(x^{i}\right)$ will be derived below. Thus, if $L(t)=1$, we have

$$
\xi^{i}=h\left(x^{i}\right) c t .
$$

These coordinates must also satisfy the equation

$$
\frac{\partial^{2} \xi^{i}}{\partial c t \partial x^{i}}=\frac{1}{c} \frac{\dot{a}}{a} \frac{\partial \xi^{i}}{\partial x^{i}}
$$

(with no implied summation over ' $\mathrm{i}$ '), so that

$$
\frac{d h\left(x^{i}\right)}{d x^{i}}=\frac{1}{c} \frac{\dot{a}}{a}\left(\frac{d h\left(x^{i}\right)}{d x^{i}} c t\right),
$$

which means that $a(t)$ must again satisfy the equation

$$
\frac{1}{c} \frac{\dot{a}}{a} c t=1,
$$

reproducing the result in Equation (8).

Finally, from the $\mu=\nu=1$ component in Equation (4), we get

$$
h\left(x^{i}\right)=h_{0} e^{x^{i} / c t_{0}},
$$

where $h_{0}$ is an integration constant, so that a redefinition of the spatial scale,

$$
\chi^{i} \equiv h_{0} c t_{0} e^{x^{i} / c t_{0}},
$$

allows us to formally write

$$
\xi^{i}=a(t) \chi^{i} .
$$

Together, the coordinates $\xi^{\mu}$ reveal that choosing the lapse function $L(t)=1$ in the comoving frame necessarily constrains the expansion factor to be uniquely $a(t)=\left(t / t_{0}\right)$ (or $a=$ constant for Minkowski), and the locally inertial frame is then simply the Hubble flow with $\xi^{\mu}=\left(c t, a \chi^{1}, a \chi^{2}, a \chi^{3}\right)$. 


\section{DISCUSSION AND CONCLUSION}

The conclusion drawn from Equations (5)-(8) appears to be unavoidable. The Local Flatness Theorem derives from foundational principles in GR, notably the concept of equivalence that allows one to account for spacetime curvature with reference to a local inertial frame. Whereas velocity is relative in GR, acceleration is absolute, so the time measured by an accelerated observer must be dilated relative to that in a free-falling frame. This effect is typically ignored in FLRW, however, regardless of whether or not the expansion is accelerated, with the belief that one may always redefine the time coordinate in order to cast the metric in standard form with $g_{t t}=1$. But in this paper we have proven that the existence of a locally inertial frame, and the strict relationship (Eq. 4) that must be respected between its coordinates and those of the comoving frame, absolutely constrain the expansion factor $a(t)$ that can be used with the lapse function $L(t)=1$. Not surprisingly, only an equation of state yielding a non-accelerating Universe is permitted by the Local Flatness Theorem.

Why then does the standard model of cosmology $(\Lambda \mathrm{CDM})$, with an empirically inferred blend of baryonic and cold dark matter, radiation and a cosmological constant, appear to be consistent with $L(t)=1$, even though it produces a complicated redshift-dependent equation of state and $\ddot{a} \neq 0$ ? In spite of the apparent flexibility afforded the expansion dynamics by its highly parameterized formulation, $\Lambda \mathrm{CDM}$ ironically adheres to the constraint we have derived when we view its evolution averaged over a Hubble time. Even though its changing constituents could have produced all manner of acceleration or deceleration, the observations are telling us that the Hubble radius today, $R_{\mathrm{h}} \equiv c / H_{0}$, in terms of the Hubble constant $H_{0}$, equals $c t_{0}$ within the measurement error. This equality is precisely what we would have measured if $a(t)=\left(t / t_{0}\right)$ all along [5, 9-12]. Clearly, the equality $R_{\mathrm{h}}=c t_{0}$ is far more than a coincidence because, in $\Lambda \mathrm{CDM}$, it could have occurred only once in its entire history, and we are seeing it right now, just when we happen to be looking.

Given the impact of our argument in this paper, it would be sensible to simply impose the zero active mass condition in $\Lambda \mathrm{CDM}$ and test whether or not it is supported by the observations. But this exercise has in fact already been done using over 25 different kinds of data, both at low and high redshifts, with integrated distances and times, and also with a differential rate of expansion. In every instance, the outcome has shown that the introduction of the equation of state $\rho+3 p=0$ into the framework of $\Lambda$ CDM reduces the tension between 
the measurements and theoretical predictions (see, e.g., Table 1 in ref. [13]). In other words, the emerging disparity between the predictions of standard $\Lambda$ CDM and the observations has actually been calling for a re-assessment of its foundational constructs. In this paper we have proven that a crucial ingredient - the zero active mass condition - cannot be ignored if the standard model continues to base its dynamical evolution on the FLRW metric.

Going forward, there are many serious consequences of this work, notably its impact on inflation. A universe with $a(t)=\left(t / t_{0}\right)$ does not have a horizon problem $[14,15]$, so it can do without an inflationary phase to account for the uniformity of the microwave background across the sky. This may be the reason why, even after four decades of development, a complete theory of inflation $[16,17]$ continues to be elusive $[18,19]$. Our conclusion in this paper suggests that inflation was not required, solving the long-standing horizon problem elegantly and simply. Novel high-precision tests are underway [20], so we may have a definitive observational confirmation of this result within a matter of years.

Acknowledgements: I acknowledge Amherst College for its support through a John Woodruff Simpson Fellowship.

[1] J. R. Oppenheimer \& G. M. Volkoff, Phys. Rev., 55 (1939) 374.

[2] G. C. McVittie, Ap.J., 140 (1964) 401.

[3] C. W. Misner \& D. H. Sharp, Phys. Rev., 136 (1964) 571.

[4] I. H. Thompson \& G. F. Whitrow, MNRAS, 136 (1967) 207.

[5] F. Melia, Frontiers of Phys., 11 (2016) id. 118901.

[6] A. Friedmann, Zeitschrift für Physik, 10 (1923) 377.

[7] Planck Collaboration, A\&A, in press (2018) (eprint arXiv:1807.06209).

[8] S. Weinberg, "Gravitation and Cosmology: Principles and Applications of the General Theory of Relativity," (1972) New York: Wiley.

[9] F. Melia, "The Edge of Infinity-Supermassive Black Holes in the Universe," (2003) Cambridge: Cambridge University Press.

[10] F. Melia, MNRAS 382 (2007) 1917

[11] F. Melia \& M. Abdelqader, IJMP-D, 18 (2009) 1889.

[12] F. Melia \& A.S.H. Shevchuk, MNRAS, 419 (2012) 2579. 
[13] F. Melia, MNRAS, 464 (2017) 1966.

[14] F. Melia, A\&A, 553 (2013) A76.

[15] F. Melia, EPJ-C Letters, 78 (2018) 739.

[16] A. H. Guth, PRD, 23 (1981) 347.

[17] A. Linde, PLB, 108 (1982) 389.

[18] A. Ijjas, P. J. Steinhardt \& A. Loeb, PLB, 723 (2013) 261.

[19] A. Ijjas, P. J. Steinhardt \& A. Loeb, PLB, 736 (2014) 142.

[20] F. Melia, MNRAS Letters, 463 (2016) L61. 